\title{
Intracranial Epidermoid Cyst Including Elements of Old Hematoma
}

\author{
-Case Report- \\ Hideo Tsurushima, Takao Kamezaki, Yuji TOMONO*, and Tadao NOSE \\ Department of Neurosurgery, Ibaraki Seinan Medical Center Hospital, Ibaraki; \\ *Department of Neurosurgery, Institute of Clinical Medicine, \\ University of Tsukuba, Tsukuba, Ibaraki
}

\begin{abstract}
A 70-year-old female presented with symptoms of right-sided trigeminal neuralgia. Computed tomography showed a high-density mass in the prepontine cistern without enhancement. Magnetic resonance (MR) imaging showed the mass as heterogeneous with variable but largely high-signal intensity on $T_{1}$-weighted images and low-signal intensity on $T_{2}$-weighted images. At surgery, the lesion was found to be an epidermoid cyst filled with old blood and lipid debris. The high-signal intensity on the $\mathrm{T}_{1}$-weighted images may reflect lipid or methemoglobin with the low intensity on $\mathrm{T}_{2}$-weighted images representing hemosiderin. Most intracranial epidermoid cysts appear as low-intensity lesions on $T_{1}$ weighted images and high-signal intensity on $\mathrm{T}_{2}$-weighted images. Typical $\mathrm{MR}$ imaging findings are neither specific for nor constant with epidermoid cysts, requiring critical differential diagnosis.
\end{abstract}

Key words: intracranial epidermoid cyst, magnetic resonance imaging, hematoma

\section{Introduction}

Intracranial epidermoid cysts are reported to constitute $0.2 \%$ to $1.8 \%$ of intracranial tumors. ${ }^{5,6,11,13,15)}$ Intracranial epidermoid cysts are congenital lesions, which most often occur in the cerebellopontine angle or the parasellar region., ${ }^{213-16)}$ The cyst walls are composed of keratinizing stratified squamous epithelium without skin appendages. The cyst contents include soft, flaky, pearly material resulting from desquamation of the cyst wall, keratin, and cholesterol crystals. ${ }^{8,12\}}$ Most epidermoid cysts appear as low density on computed tomography (CT) scans, ${ }^{3)}$ with occasional instances of isodensity ${ }^{11]}$ or high density. ${ }^{10)}$ CT densities greater than the hypodense attenuation value of lipid result from the excess of nonlipids such as keratin over the main lipid, cholesterol. ${ }^{13)}$ Magnetic resonance (MR) imaging is useful for visualizing detailed tumor localization and may permit some degree of chemical or physical characterization. ${ }^{8,17]} \mathrm{T}_{1}$-weighted MR imaging shows areas of low-signal intensity and $\mathrm{T}_{2^{-}}$ weighted MR imaging shows high-signal inten-

Received March 19, 1997; Accepted August 5, 1997 sity. ${ }^{15,16]}$ We present a patient with an intracranial epidermoid cyst which displayed signal intensities largely opposite to this classical pattern.

\section{Case Report}

A 70-year-old female presented with symptoms of right trigeminal neuralgia which began in late October 1996. She was admitted on November 2, 1996 with no other neurological abnormalities. She had undergone a right suboccipital craniectomy at another hospital about 30 years previously, but no specific clinical information was available. CT showed a homogeneous high-density mass in the prepontine cistern, which was not enhanced by administration of contrast medium (Fig. 1). $\mathrm{T}_{1}$-weighted MR imaging showed a heterogeneous mass with areas of high- and low-signal intensity (Fig. 2A, B), but no enhancement with gadolinium-diethylenetriaminepenta-acetic acid. $\mathrm{T}_{2}$-weighted $\mathrm{MR}$ imaging showed a homogeneous mass with low-signal intensity (Fig. 2C, D).

After treatment to improve thrombocytopenia associated with cervical lymphangitis, the mass was removed on December 11, 1996. A right suboccipital 


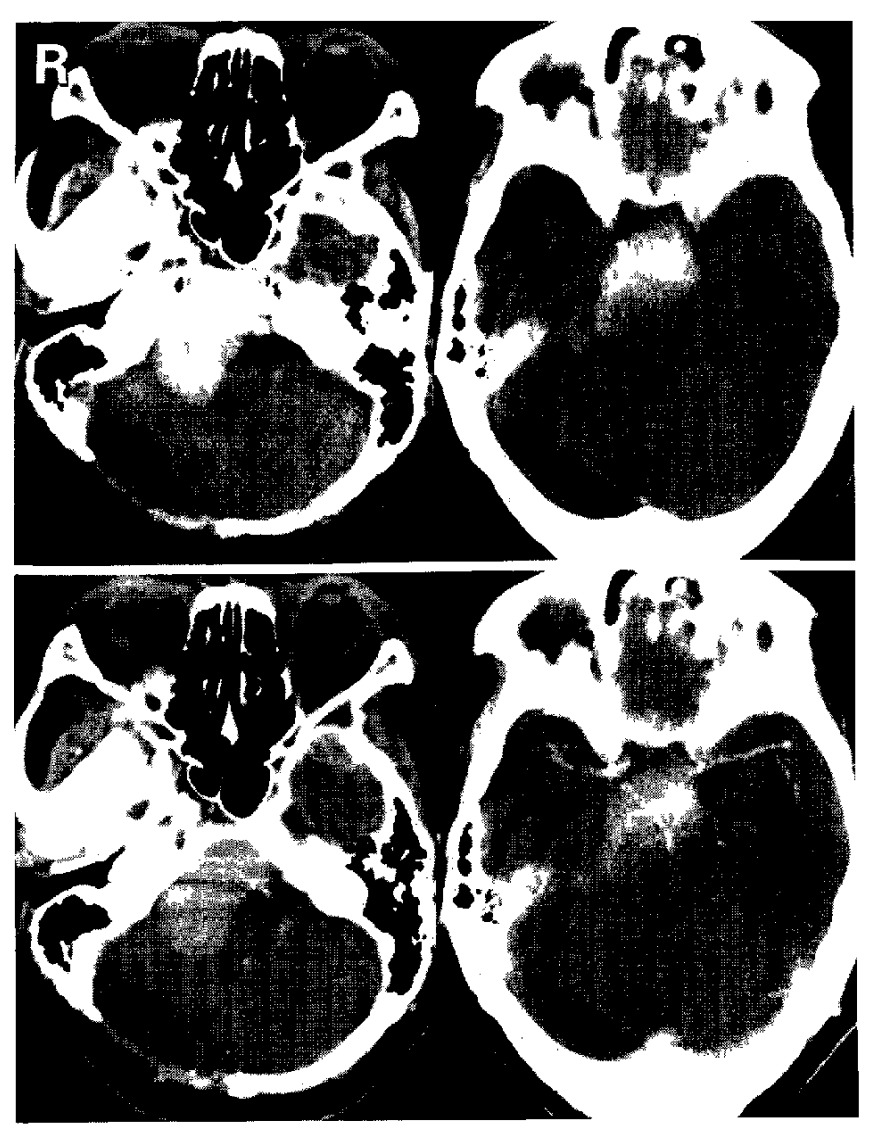

Fig. 1 Computed tomography (CT) scans showing an epidermoid cyst in the right cerebellopontine cistern. CT scans (upper row) showing a high-density mass displacing the brain stem, and evidence of prior craniectomy. CT scans with contrast medium (Iower row) showing no enhancement.

reopening exposed a cyst with a white wall in the prepontine cistern beyond the cranial nerve VIIVIII complex. The cyst wall of the lesion was punctured, revealing sludgy contents resembling machine oil, suggesting lipid debris and old blood. The sludge was sucked off and as much of the cyst wall as possible was removed. The cyst wall adhered to the brain stem and all of the cyst could not be removed.

After surgery, her symptoms were relieved without complications. Histological examination of the specimen revealed keratinizing, stratified squamous epithelium without skin appendages and hemosiderin in the cyst wall with Berlin blue stain (Fig. 3). The histological diagnosis of the lesion was epidermoid cyst. It was suspected that this epidermoid cyst contained old blood, since hemosiderin was confirmed.

\section{Discussion}

Epidermoid cysts usually show signal intensities intermediate between those of the cerebrospinal fluid and the brain on $\mathrm{T}_{1}$-weighted MR images, and a signal intensity greater than that of brain on $\mathrm{T}_{2}$-weighted MR images. ${ }^{15,16)}$ However, intensities on MR images may vary and in some cases the detailed findings with epidermoid cysts have not been reported. ${ }^{8 j}$ The varying intensities on $T_{1}$ and $T_{2}$-weighted images reflect the differences in chemical components of the epidermoid tissue and cyst contents. ${ }^{8]}$ We believe that the high density observed on CT scans was due to old hematoma, the high-signal intensity on $\mathrm{T}_{1}$-weighted $\mathrm{MR}$ images was due to lipid and methemoglobin, and the low-signal intensity on $\mathrm{T}_{2}$-weighted images was due to accumulation of hemosiderin in our case. These findings suggest the possibility of bleeding into the cyst, but this occurrence must be distinctly uncommon given the avascular nature of these lesions. There are four previously published cases of intradural epidermoids with elements of hematoma. ${ }^{1,4,7,9)}$ Hasegawa et al. ${ }^{7)}$ surmised bleeding into the cyst from the fibrous nodule of the cyst wall, which contained vessels. However, the mechanism of the hemorrhage in our case is uncertain.

Despite the important anatomic information regarding the lesion and the brain provided by MR imaging, MR imaging findings of epidermoid cysts are neither specific nor constant. MR imaging has not eliminated the need for a thoughtful, critical approach to differential diagnosis.

\section{Acknowledgment}

The authors would like to thank Mr. S. Kouya for his technical support.

\section{References}

1) Abou-Samra M, Marlin AE, Story JL, Brown WE: Cranial epidermoid tumor associated with extradural hematoma. Case report. J Neurosurg 53: 574-575, 1980

2) Berger MS, Wilson CB: Epidermoid cysts of the posterior fossa. J Neurosurg 62: 214-219, 1985

3] Davis KR, Roberson GH, Taveras JM, New PEJ, Trevor R: Diagnosis of epidermoid tumor by computed tomography. Radiology 119: 347-353, 1976

4) Dunn RC, Archer CA, Rapport RL, Looi LM: Unusual CT-dense posterior fossa epidermoid cyst. Case report. J Neurosurg 55: 654-656, 1981

5) Eekhof JLA, Thomeer RTWM, Bots GTAM: Epidermoid tumor in the lateral ventricle. Surg Neurol 23: 189-192, 1985 


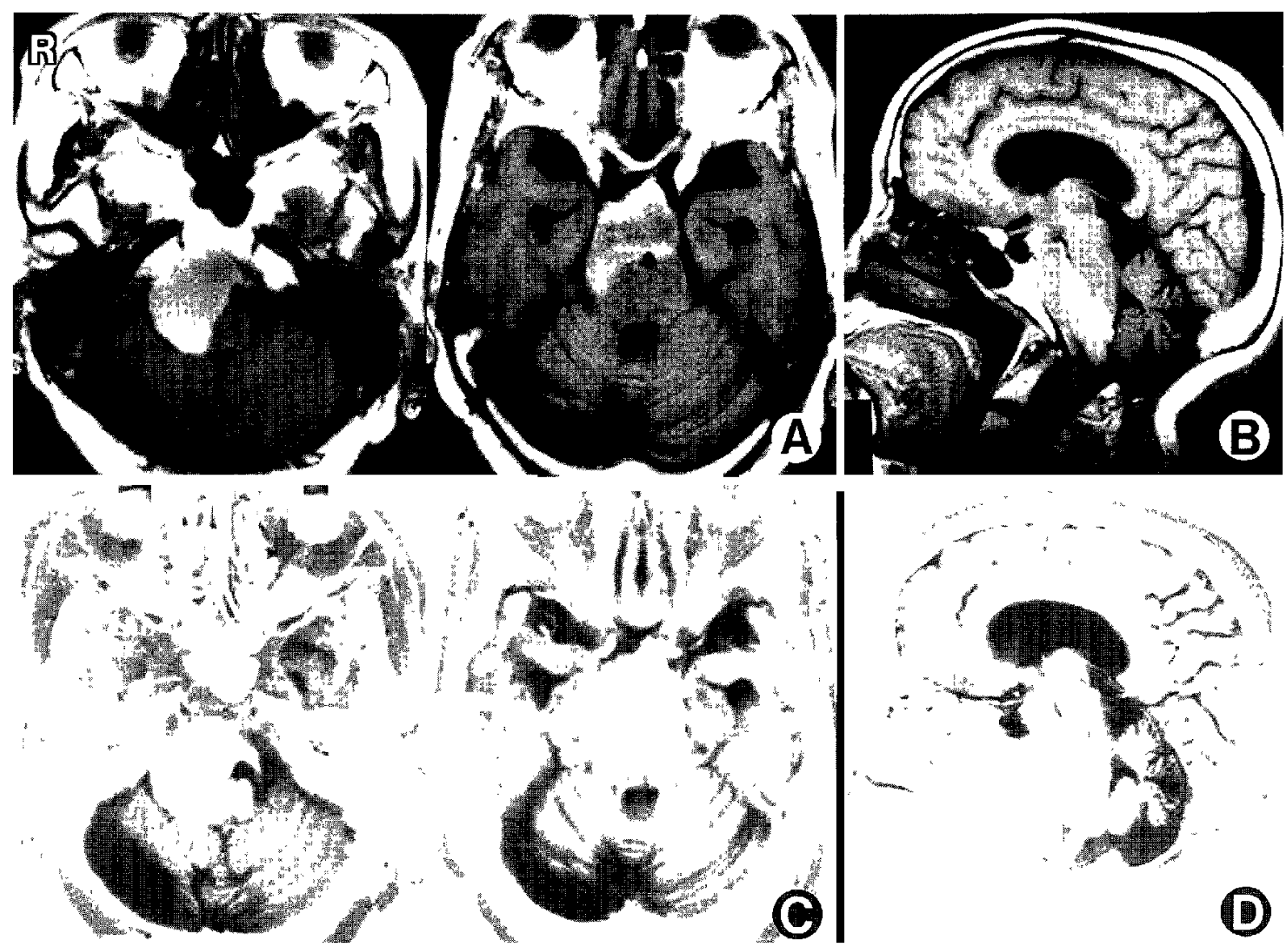

Fig. 2 Magnetic resonance images showing the intracranial epidermoid cyst. Axial (A) and sagittal (B) $T_{1}$-weighted images showing a high-intensity area. Axial (C) and sagittal (D) $T_{2}$-weighted images showing a low-intensity area.

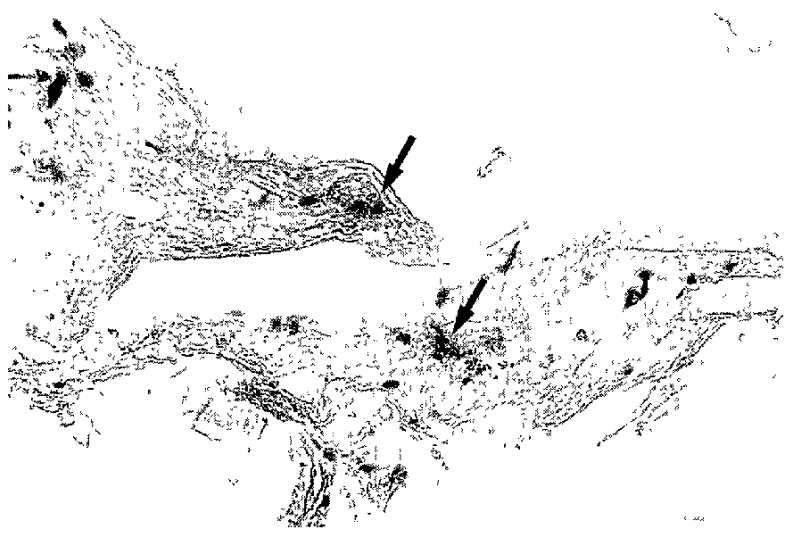

Fig. 3 Photomicrograph of the cyst wall showing hemosiderin (arrows). Berlin blue stain, $\times 200$.

6) Fiume D, Gazzeri G, Spallone A, Santucci N: Epidermoid cysts of the fourth ventricle. Surg Neurol 29:
$178-182,1988$

7) Hasegawa H, Bitoh S, Nakata M, Fujiwara M, Yasuda $\mathrm{H}$ : Intracranial epidermoid mimicking meningioma. Surg Neurol 15: 372-374, 1981

8) Ishikawa $M$, Kikuchi $H$, Asato $R$ : Magnetic resonance imaging of the intracranial epidermoid. Acto Neurochir (Wien) 101: 108-111, 1989

9) Lunardi P, Rizzo A, Guidetti G: Unusual CT-dense transtentorial epidermoid cyst. Neurochirurgia (Stuttg) 31: 219-221, 1988

10) Maehara $F$, Ishii $K$, Takahashi $S$, Goto $K$, Okudera $T$, Fukushima T, Tomonaga M: [High-density intracranial epidermoid tumors: Computed tomographic findings in 3 cases]. CT Kenkyu 5: 157-163, 1983 (Jpn)

11) Obrador S, Lopez-Zafra JJ: Clinical features of the epidermoids of the basal cistern of the brain. J Neurol Neurosurg Psychiatry 32: 450-454, 1969

12] Ogawa T, Sekino H, Fuse T, Nakamura N: [Multiple intracranial epidermoids located in the brain stem and the middle cranial fossa. Case report]. Neurol Med Chir (Tokyo) 25: 393-397, 1985 (Jpn, with Eng abstract)

13) Russel DS, Rubinstein LJ: Pathology of Tumors of the 
Nervous System, ed 5. London, Edward Arnold, 1989, pp 690-695

14) Ulrich J: Intracranial epidermoids: A study on their distribution and spread. J Neurosurg 21: 1051-1058, 1964

15) Yamakawa $K$, Shibata N, Genka S, Manaka S, Takakura K: Clinical course and surgical prognosis of 33 cases of intracranial epidermoid tumors. Neurosurgery 24: 568-578, 1989

16) Yasargil MG, Abernathey CD, Sarioglu AC: Microneurosurgical treatment of intracranial dermoid and epidermoid tumors. Neurosurgery 24: 561-
567,1989

17) Yoshizato K, Kai Y, Kuratsu J, Ushio Y: Intramedullary epidermoid cyst in the brain stem: Case report. Surg Neurol 45: 537-540, 1996

Address reprint requests to: H. Tsurushima, M.D., Department of Neurosurgery, Ibaraki Seinan Medical Center Hospital, 2190 Sakai-machi, Sashima-gun, Ibaraki 306-04, Japan. 